\title{
Enriched Environment Procedures for Rodents: Creating a Standardized Protocol for Diverse Enrichment to Improve Consistency across Research Studies \\ Bruce C. Harland ${ }^{1}$ and John C. Dalrymple-Alford ${ }^{2,3, *}$
}

${ }^{1}$ Department of Pharmacy, University of Auckland, Auckland, New Zealand; ${ }^{2}$ School of Psychology, Speech and Hearing, University of Canterbury, Christchurch, New Zealand; ${ }^{3} \mathrm{New}$ Zealand Brain Research Institute and Brain Research, Christchurch, New Zealand

*For correspondence: john.dalrymple-alford@canterbury.ac.nz or ic.dalrymple.alford@gmail.com

[Abstract] Exposure to environmental enrichment has beneficial effects on learning and memory, diverse neurobiological effects, and promotes recovery of function after brain injury. The effect of enrichment is produced by a combination of increased social interaction, physical activity, spatial complexity, and novelty. Procedures in the literature have, however, been idiosyncratic with poor consistency in the manner or extent to which protocols provide consistent enrichment. We provide an environmental enrichment protocol that can be easily replicated with minor details determined locally so that animals across cohorts and cages all experience a comparable level of enrichment. Procedures are outlined to generate and use a daily pool of suitably varied objects using a standardized format, with objects systematically varied up to a 40-day continuous period. Together with using a large group of rats in a suitably-sized cage, and regular shifting of the position of food and water and cage location, these procedures have produced robust effects in different laboratories and rat strain, thereby improving comparisons within and across laboratories. Non-enriched comparisons can vary, but typically would include grouped animals in standard laboratory housing without objects and with stable food and water locations. Enrichment is a safe non-pharamacological tool to examine behavioral and neurobiological processes in animal models of the lifespan, brain dysfunction and injury.

Keywords: Enrichment, Environmental enrichment, Enriched environment, Recovery of function, Cognitive reserve, Novel objects, Social stimulation, Rat, Mouse

[Background] Enriched environments are a proven therapeutic tool to ameliorate cognitive and affective deficits associated with age-related decline and after many types of brain injury (van Praag et al., 2000; Will et al., 2004; Nithianantharajah and Hannan, 2006; Pang and Hannan, 2013; Alwis and Rajan, 2014; Hannan, 2014). This includes improved cognitive reserve, recovery from relatively diffuse brain injury, such as stroke, traumatic brain injury and various neurodegenerative diseases (Johansson, 2004; Pang and Hannan, 2013; Alwis and Rajan, 2014; Hannan, 2014), as well as from acute localized lesions in cortex, hippocampal formation and thalamus (Will and Kelche, 1992; Will et al., 2004; Harland et al., 2014; Dalrymple-Alford et al., 2015). These effects are likely to be mediated by increased activation of multiple brain networks, including their recruitment during behavioral tasks. Neurobiological effects range from the size and morphology of brain regions to the survival and complexity of neurons, adult neurogenesis, enhanced cell excitability, synaptic plasticity, place cell function, and a wide array 
of neuroprotective molecular responses that reflect multiple genetic processes, including geneenvironment interactions, neuroinflammation and trophic factors (Rampon et al., 2000a and 2000b; Will et al., 2004; Leger et al., 2012; Bonaccorsi et al.,2013; Briones et al., 2013; Eckert and Abraham, 2013; Alwis and Rajan, 2014; Hirase and Shinohara, 2014; Bilkey et al., 2017; Kempermann, 2019; Ohline and Abraham, 2019). These benefits of enrichment are thought to arise from a combination of increased exercise, socialization, and novelty, producing stronger effects than each of these components administered individually (Einon et al., 1980; Will et al., 1986; Olson et al., 2006; Cracchiolo et al., 2007; Sozda et al., 2010). Environmental enrichment is often used as general 'cognitive treatment' and produces behavioral and cognitive enhancements in young and old intact or 'sham-operated' rats (Gonzalez-Ramirez et al., 2014; Harland et al., 2014). Laboratories that study spatial navigation, memory, or neurogenesis, often raise experimental animals in enriched environments as a cognitive enhancer, for example, groups that study in vivo neuronal electrophysiology (e.g., Moser group, Kavli Institute, Norway; Jeffery group, University College London, United Kingdom).

An enriched environment for rats or mice in a laboratory setting generally includes a moderate (1012) group of animals housed in a large cage in which they are exposed to stimulation far beyond that afforded by the standard, generally barren laboratory cage that often houses 3 to 6 animals (Simpson and Kelly, 2011). Explicit environmental stimulation is provided by adding multiple objects within the enrichment cage. However, these objects are sometimes infrequently or randomly varied in many studies. More consistent enrichment should regularly introduce new objects and new configurations, preferably on a daily basis. This more systematic approach provides informal, but varied and multisensory cognitive stimulation and learning, together with physical and social experiences. Unlike natural (feral) conditions, laboratory enrichment establishes a safe complex environment that has relatively stable social conditions but in which the inanimate physical conditions are frequently changing. Environmental enrichment procedures, such as the number of rats per cage, cage equipment and space, and especially the type and frequency of objects, have often varied markedly across enrichment studies (Simpson and Kelly, 2011). A range of different exposure periods has been implemented, with the majority of studies focusing on enrichment for 30 or 40 days. Periods as short as 4 days or noncontinuous exposures of just several hours a day have also been used (Kolb and Gibb, 1991; Wallace et al., 1992; Bindu et al., 2005). The current protocol outlines a systematic method to produce standardized continuous enrichment paradigms for use with rats (or mice) to allow different cohorts of animals to experience comparable enrichment that can be easily replicated in most laboratories to achieve more consistent enrichment procedures. A rigidly standardized protocol across all labs is not feasible, but our protocol provides a clear guide of how a greater degree of standardized enrichment can be achieved than is currently the case. It describes a systematic approach in the frequency, variation and placement of objects to standardize this aspect of the enrichment procedure across experiments within any given lab and to encourage greater similarity across laboratories with respect to object-based enrichment protocols. 


\section{Materials and Reagents}

\section{Bedding}

Bedding should be changed at least once per week; for us this was once per week just after the cages were washed. We used Natures Flame pellet fuel (Nature's Flame Limited, New Zealand 1PSI) for bedding in standard (control) cages, which breaks down into sawdust over a few days. The same bedding, but already in sawdust form, was used in the enrichment cages, to help facilitate the positioning of objects; this was created by soaking and breaking up the Natures Flame pellets in a little water and then leaving them to dry. Presoaking of the bedding material for the standard-housed rats could also be used, but the additional technician time required for the standard cages is not necessary. Any suitable laboratory-standard animal bedding is suitable from your supplier and this does not need to be varied between enriched and standard caging.

2. Rats

We have used male hooded PVGc rats (e.g., Harland et al., 2014), as well as female hooded PVGc rats, with this protocol, which were bred in-house in our Facility. Only single-sex housing is used in the enrichment literature. If female rats are used, we have not found it necessary to control for the estrus cycle; this decision may depend on the particular study, especially where hormonal changes are relevant. Our protocols have been shown to be effective in Long-Evans rats (Ali et al., 2017). Any persistently aggressive animal should be removed, and more frequently monitored if a given strain is known in this regard. Our rat strain was originally supplied by an Animal Resources Centre in Australia. A wide variety of different rat (and mouse) strains have been used in the literature; direct comparisons of rat strain for enrichment effects are uncommon. Although it is common in the current literature to provide the weight of rat, for enrichment the more relevant variable is the actual age of the animals used (an estimate of age on arrival can be provided by your supplier if in-house breeding is not employed). The age of rat used would be determined by the specific experimental questions under study. In standard laboratory housing conditions, some strain of rat (e.g., Long-Evans; some albino strains) can show evidence of obesity after young adulthood (Tordoff et al., 2008). Obesity in these strains needs to be countered by advance planning of mild food restriction when young adults and this may require comparisons of weight gain across the two housing conditions. Enriched rats are generally lighter than standard-housed rats, but marked differences in weight should be avoided in our view by mild food restriction in both groups.

3. Enrichment objects

A large collection of objects with at least four copies of each-see Step A2 for guide on collecting suitable objects. Only clean objects should be introduced.

4. Clips

An assortment of clips to connect enrichment objects to each other or to the walls of the cage. For example, we used: Medium fold-back paper clips (e.g., Medium Binder Clip, Staples, catalog 
number: 103549) and S Hooks (e.g., 1" S Hook, Staples, catalog number: 272145).

5. Detergent

To clean enrichment objects daily. We used Pyroneg powder (2-3 grams per litre; Thermo Fisher, catalog number: DIVHH13231).

6. Wooden blocks

Non-toxic small hard-wood blocks from your local supplier $(8.5 \times 9.4 \mathrm{~cm}$, and $1.5 \mathrm{~cm}$ thick or similar), are used for rats to gnaw on after initially sterilized. These blocks minimize any damage to objects. Any contaminant-free wooden blocks would be suitable (e.g., Wood Gnawing Blocks, Bio-Serv, catalog number: K3512).

\section{Equipment}

1. Large enrichment cages

We used locally built wire-mesh hot-dip galvanized cages to house 10-12 rats (maximum of 12 in our case). The cage was $85 \mathrm{~cm}$ long by $60 \mathrm{~cm}$ wide by $30 \mathrm{~cm}$ high, with a solid metal floor and two hinged mesh lids. Figures 1A-1B show our enrichment cage. Figures $1 \mathrm{C}-1 \mathrm{D}$ shows two groups of rats participating in the enriched environment (the environment changes daily). Enrichment cages can be constructed by local manufacturers (as in our case) or custom built by cage suppliers. Another option could be to use suitable commercially available cages for pet rats, hamsters or birds. Multi-level cages may be used, but these are regarded as a modification to standardized enrichment protocols. The size of cage and number of rats should accommodate the strain being used and local conditions and requirements. The floor area per animal for the enrichment cage (height $x$ width of floor divided by number of animals; $425 \mathrm{~cm}^{2}$ in our protocol with 12 male rats) is a little greater than the floor area per animal in the standard laboratory cages $\left(375 \mathrm{~cm}^{2}\right.$ in our protocol with 4 male rats). The minimum floor space per rat based on weight should follow the National Research Council: Guide for the use and Care of Laboratory Animals (2011) (Reference 23) guidelines: e.g., for rats $<100 \mathrm{~g}$ floor area per animal $>109.6 \mathrm{~cm}^{2}$, for rats $<200 \mathrm{~g}$ floor area per animal $>148.35 \mathrm{~cm}^{2}$, for rats $<300 \mathrm{~g}$ floor area per animal $>187.05 \mathrm{~cm}^{2}$, for rats $<400 \mathrm{~g}$ floor area per animal $>258 \mathrm{~cm}^{2}$, for rats $<500 \mathrm{~g}$ floor area per animal $>387 \mathrm{~cm}^{2}$, for rats $>500 \mathrm{~g}$ floor area per animal $>451.5 \mathrm{~cm}^{2}$. The guide also recommends that cages should be $<17.8 \mathrm{~cm}$ high, but we recommend $<27 \mathrm{~cm}$ high if possible, to allow rats to stand fully upright (Makowska and Weary, 2016). Very large rats (> $900 \mathrm{~g}$ ) may need even more space, such as $1000 \mathrm{~cm}^{2}$ floor area per animal and $30 \mathrm{~cm}$ cage height (see Lawlor, 1990). "Crowding" in our enrichment cages does not occur because rats have the whole area to explore and rats generally socially interact and aggregate together no matter what the size of cage provided. For example, object \#13 (Appendix 1 in Supplemental) shows a $15 \times 15$ $x 15 \mathrm{~cm}$ clear Perspex box with holes for entry/exit; there may be 2 to 6 rats who self-select to spend periods of time within this box. Within reason, social interaction is more critical than the space afforded per rat within the cage. However, rats in enrichment should be monitored for any 
overt signs of stress (behavioral changes, vocalization, chromodacryorrhea, unexpected weight loss). Plasma corticosterone response could also be monitored as an analog of stress; other researchers using our enrichment protocol reported relatively low levels of intracardial plasma corticosterone $(150 \mathrm{ng} / \mathrm{ml})$ and no differences on this measure between enriched and standard housed rats at the end of a 40-day continuous period of differential housing (see Supplementary Figure 2 from Ali et al., 2017). Small variations in cage size is unlikely to have a substantial effect on the results. Our cages were specifically designed to be able to be housed within racks in our colony rooms, so it is important to consider these kinds of restrictions when ordering cages. Cages need to be high-grade stainless steel and be able to withstand repeated exposure to an industrial (walk-through) cage washer. Alternatively, iron or steel cages can be hot-dip galvanized in zinc to make them rustproof.
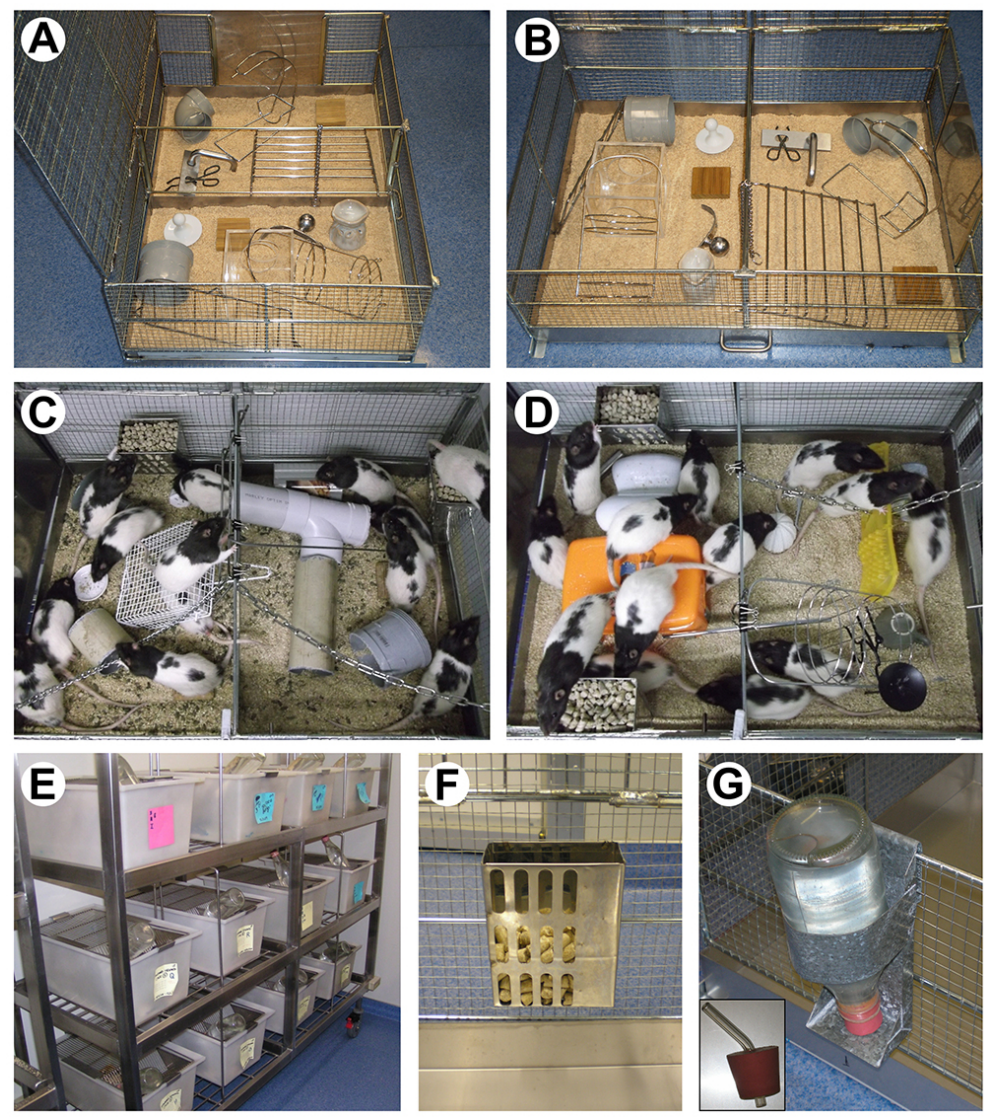

Figure 1. Examples of the enrichment setup used in our laboratory. $A$ and $B$. The enrichment cage, containing an example of the daily configuration of objects, is shown from (A) the side, and $(B)$ the front. $C$ and $D$. Rats participating in environmental enrichment in two different object configurations are shown in $(E)$. E. Example of standard cages in the colony room. F. Moveable metal food hoppers that could be attached at different heights and positions within the enrichment cages. G. Glass water bottle mounted in moveable metal holder; the bottle cap (shown in insert) was hard rubber and contained a glass spout angled into the cage. 
2. Standard cages

We used opaque (autoclavable) plastic cages to house 3-4 rats. These standard housing cages were $50 \mathrm{~cm}$ long by $30 \mathrm{~cm}$ wide by $22 \mathrm{~cm}$ high, with stainless steel grille lids. Figure $1 \mathrm{E}$ shows our standard cages on a stainless-steel rack in a colony room. Any kind of regular laboratory cage, which houses 2-6 animals (we usually have 3 or 4), would be suitable for use as the "standard" housing condition. If there is a local preference that one or two objects be placed in this cage and remain unchanged (other than for cleaning) we do not regard this as "enrichment".

3. Food hoppers

Figure $1 \mathrm{~F}$ shows the food hoppers allowing easy relocation of food around the inner walls of the enrichment cage (e.g., Ancare mouse or rat feeders, catalog number: MF342/RF452). Any standard laboratory rat chow is suitable to use as food for both the enriched and standard housing.

4. Metal bottle holders

Figure $1 \mathrm{G}$ shows glass bottles $(600 \mathrm{ml})$ and metal bottle holders used in our studies allowing easy relocation of water position across days (e.g., Ancare sure lock bottle holders, catalog number: $\mathrm{H}-100$ to $\mathrm{H}-102)$. Any bottle compatible with the holder would be suitable. The drinking spout should be rodent compatible (i.e., with one or more metal ball bearings to hold water contents) and long enough to protrude inside the cage wall.

5. (Optional) Light

We use a reversed light cycle in the colony room so that behavioral testing is conducted in their dark period, that is, when rats are naturally more often active. The test environment can of course have lighting, because the modern-day natural environment would also include lit areas during the true night-time period. This is a local preference issue.

Note: All equipment was cleaned once a week using an industrial rack-sized cage wash, but any suitable cleaning arrangement can be used.

\section{Procedure}

A. Preparing a systematic enrichment protocol Note: This section describes all of the steps for designing and creating an enrichment protocol.

1. Decide on enrichment parameters

a. Decide on the length of the continuous enrichment period. It is common to use 30 or 40 days, and positive molecular, cellular and behavioral effects in rodents have been shown at these enrichment durations (see Background). However, the duration of enrichment is based on the particular experimental design and specific questions. Positive effects have been demonstrated after as few as four continuous days of enrichment, or non-continuous exposures of just several hours a day.

b. Decide on the number of objects per day. We recommend 10-15 objects per day (we used 13). It is best to balance between number of objects and the remaining space within the 
cage. The objects should be distributed throughout the cage, but not restrict the movement of the rats. Our enrichment templates are in the Supplemental section and provide examples that can be followed.

c. Designate a number of days (referred to as a "cycle" of days) in which all of the objects will be used only once (i.e., the same object is never repeated for a given cage). To make things easy, this cycle should be divisible by the enrichment period (i.e., use 5 or 6 days for a 30day period; use 5 or 8 days for a 40-day period). For our 40-day enrichment protocol, we created a cycle of 6 object-days, followed by a $7^{\text {th }}$ day with 'no objects', followed by an $8^{\text {th }}$ day that exclusively used 13 PVC tubing objects combined to make a tunnel system (Step A3 contains additional information about these extra "themed" days). This gave an 8-day cycle, repeated 5 times but with explicitly different arrangements of objects per 8-day cycle for the 40-day period. See Supplemental for all of our enrichment day templates. Information about the cycles is listed in the caption below each template.

Note: Using a cycle of days ensures objects are not re-used too often and that the same arrangement of objects is never used. This is achieved without the need for unique objects every single day of a long enrichment period. Using the cycle of days is also compatible with having multiple enrichment cages running concurrently while using the same overall pool of objects (see Step B1).

d. Calculate the minimum number of objects required. That is, use the number of objects per day (for us, 13 objects) multiplied by the number of days in a cycle (for us, 6 days). So in our case, we used 13 objects per day x 6-day cycle $=78$ different objects.

2. Create a collection of enrichment objects

a. A large number of objects need to be collected which are durable enough to survive multiple exposures to rats/mice, including animals that may be on food restriction. Objects need to exhibit as much variation in their size, shape, texture, color, and physical makeup as possible (Figure 2). It is also crucial to have multiple copies of each object to allow easy recycling/simultaneous use of objects in different cages/replacement in the event of any breakages. Objects must be able to endure multiple washes with industrial grade detergents. We generally avoid enrichment objects that have any fragrance and the requirement for multiple washes with industrial-grade detergents would dissipate any object-related odors. The introduction of explicitly odorized objects may be used, but generally only if a specific experimental variation is desired. This section contains suggestions for compiling such a collection of objects as cheaply as possible. 


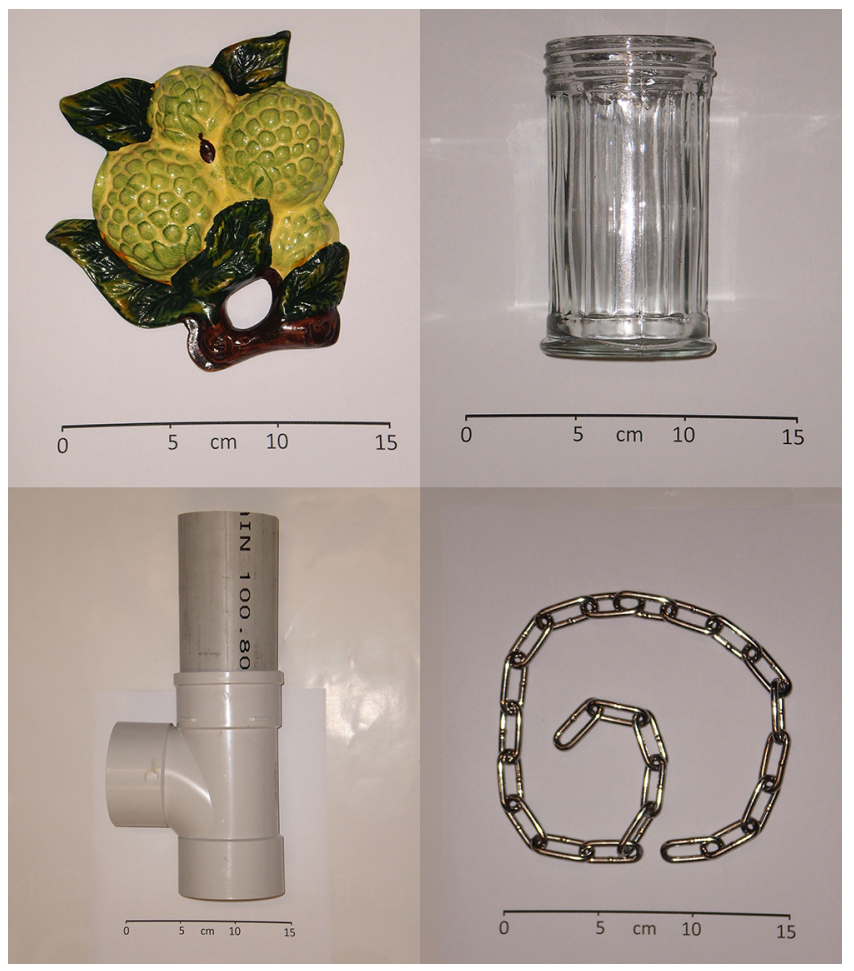

Figure 2. Examples of enrichment objects. These four example objects consist of different materials and vary in shape and texture. The 'object list' in Supplemental shows all objects used in our studies.

b. Types of enrichment objects: Good materials for objects are: hard plastics, sturdy glass, ceramic, stainless steel metal. Materials that should not be used are: any plastic thin enough to be quickly chewed, metal that can rust, paper or cardboard or any other material that can be easily chewed and consumed. We do not recommend the use of wooden objects, especially if the treatment or paint used is unknown and could be poisonous. In addition, soft wood that can be chewed and untreated hard wood that can soak up urine should not be used. Variation in size and shape is good, but avoid objects with sharp edges, and objects that are too large, heavy, or bulky for the environment size. Some thin plastic objects with no exposed edges, such as table tennis balls, are suitable.

\section{Examples of suitable object types:}

i. Ceramic statues, figurines, candle holders, bowls, cups

ii. Glass or perspex bottles, jars, paperweights, tubes, tunnels

iii. Hard plastic toys, figurines

iv. Metal chains, large screws, nuts, parts of taps or door handles, oven racks, tongs, candle-holders

v. PVC (polyvinyl chloride) tubes of different shapes, sizes

vi. Multiple versions of each object type can be used, with variations in size, color, and shape. For further examples of suitable objects see Supplemental which contains photos of all objects that we used in our study. For information on how this object list 
was made, see Step A3b. The minimum number of objects that is required is "the number of objects per day multiplied by the number of cycled days".

c. List of suitable outlets to obtain cheap objects:

i. Thrift stores: 'pound stores' (UK), 'dollar stores' (USA, New Zealand). These are particularly good for obtaining multiple copies of cheap ceramic objects, toys, figurines, jars, bottles etc.

ii. Hardware stores: great for obtaining small metal and wooden items, and PVC tubing, metal racks, e.g., 'Home Depot' (USA), 'Bunnings' (New Zealand).

iii. Discount department stores or stationary stores: items generally more expensive than thrift stores, e.g., 'Walmart' (USA), 'Argos' (UK), 'Warehouse' (New Zealand).

iv. Scrap/recycle/second-hand stores: great to find cheap intact household metal/plastic items, however, harder to find identical multiple copies of objects.

3. Create Enrichment day templates

Templates should be created for each enrichment day detailing the set of objects used for that day and their start configuration within the cage. Using templates allows these configurations to be accurately reproduced, even by other experimenters. Templates show the start configuration of objects within the cage before rats are introduced to the cage. Figure 3 shows an example template; additional example templates are shown in Supplemental. Ideally, each enrichment template will include a list, with images, of all objects used for a given day's configuration. It will also include images of the configuration of objects within the cage preferably from different angles to enable accurate positioning of objects. 


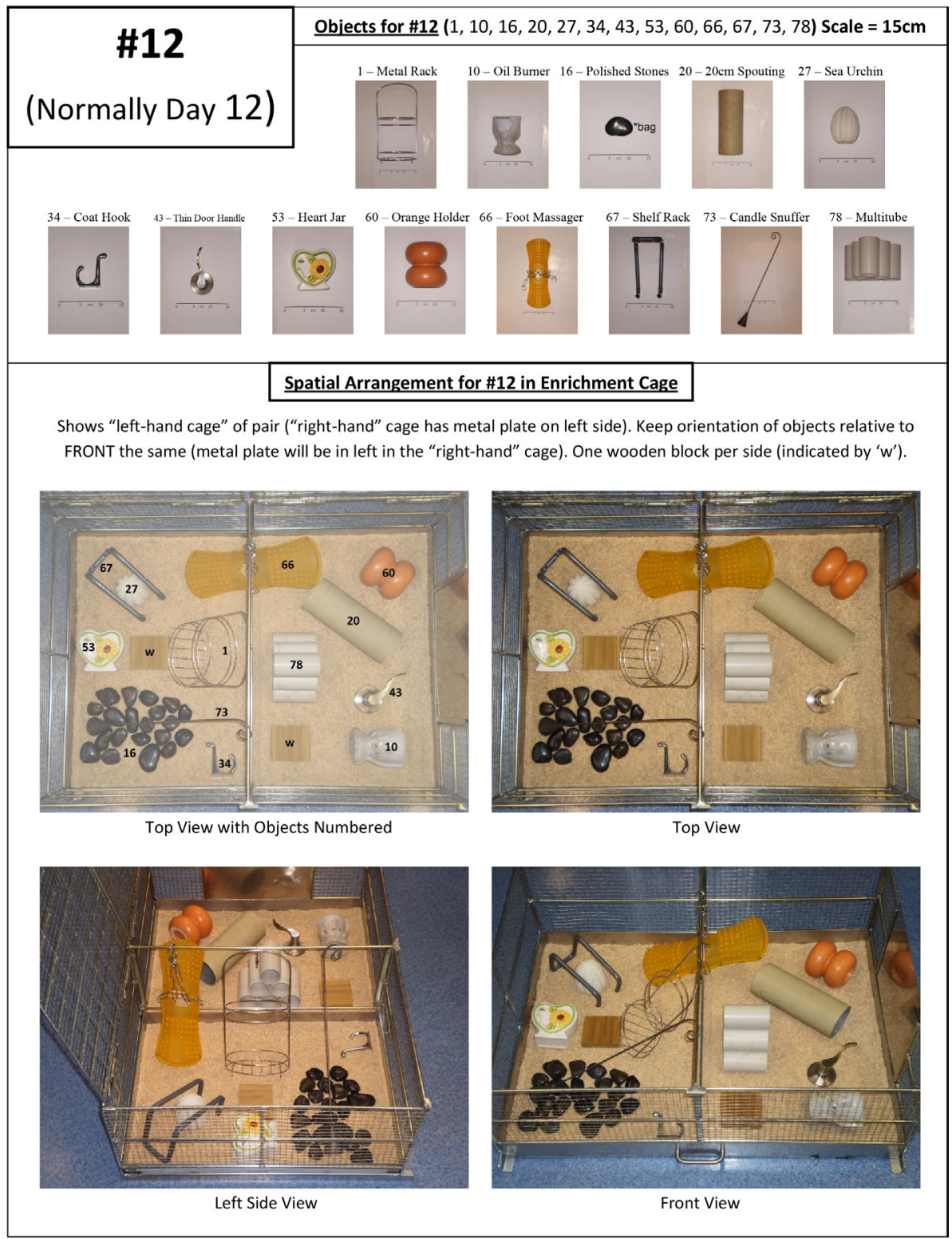

Figure 3. Example of enrichment template. An object list at the top designates the images, ID numbers, and names of all 13 objects used in the configuration (scale bars $=15 \mathrm{~cm}$ ). Four images below this show the setup position of objects in the cage from different viewpoints.

a. First, create a database of objects, containing a photo and ID number for each object. Place a ruler or scale bar on a clear horizontal surface and take digital photographs from above each object (Figure 2). Transfer 15-20 images per page into a word document (Microsoft Word or Apache OpenOffice Writer). Assign each object a unique ID number, and unique name (to describe that object in 1-3 words).

Note: Inclusion of a $15 \mathrm{~cm}$ scale bar facilitates a permanent record of the empirical size of each object and their relative size.

b. Set up and take photos of the configurations for every enrichment day. The easiest way to achieve this is to create templates for each "cycle" (in which each object is used once) and 
then move on to create templates for the next cycle. First, set-up the empty enrichment cage (no rats, but containing bedding) on the floor or on a low table or bench in a well-lit room. Divide the objects into groups containing the specified 'number of objects per day'; these initial groups of objects will make up the templates for the first cycle. For each group of objects, set them up inside the enrichment cage. Place objects so that they are arranged in diverse ways. Use suitable clips to attach objects to the walls of the cage and suspend some to incorporate the vertical space within the cage. Once a group of objects is set up, take high quality photographs from several angles so that the arrangements can be easily repeated. For example, we used an overhead photo, and $\sim 45^{\circ}$ angle photos of the cage from the front and side (see Figure 3). Now remove those objects, repeat the process with the next group of objects until unique object setups have been created and photographed for each day for the first cycle. An important step is to recombine the objects into new groups containing the specified 'number of objects per day' and repeat the process to create the $2^{\text {nd }}, 3^{\text {rd }}$, and so on, cycles, until you have created unique configurations for every day of the enrichment period. For an example of this process, see our enrichment templates in Supplemental; the five cycles we used for our enrichment (templates 1-6, 9-14, 17-22, 25$30,33-38$ ), in which every object was used once, were made using the method described above.

c. Optional: Include additional 'themed' days at the end of each cycle. For example, every $7^{\text {th }}$ enrichment day we had a 'no objects' day in which the animals were introduced to an empty enrichment cage (containing just the bedding and food/water). This was to give the animals a different experience, and to enhance subsequent 'object days. Every $8^{\text {th }}$ enrichment day, the 13 objects consisted of PVC tubing pieces connected to make unique tunnel systems. A different configuration of the PVC tubes was used across cycles. The tunnels were designed to have entry/exits facing many different directions within the cage to promote spatial learning. These PVC tubing pieces were exclusively used for these configurations, although the general object list included several tubes/tunnels as well. Another possibility would be to use LEGO Duplo bricks (or any other durable plastic brick large enough not to be consumed) to make unique walls and structures.

d. After the first cycle, ensure no object is reintroduced for at least 2 days. When re-grouping the objects into new unique combinations for the second cycle, make sure that at least 2 groups contain none of the objects from the last 2 days of the previous cycle and use these groups as your $1^{\text {st }}$ and $2^{\text {nd }}$ days for the second cycle. Similarly, when re-grouping objects to create configurations for the third cycle, ensure at least 2 groups contain none of the objects from the last 2 template days of the second cycle and use these groups as your $1^{\text {st }}$ and $2^{\text {nd }}$ days for the third cycle. Repeat this same process for each cycle. Because we included 2 additional themed days at the end of each cycle ('no object day' and 'tunnels only day') this meant no single enrichment object was reintroduced for at least 4 days. 


\section{B. Running an enrichment protocol}

This section describes all of the steps and procedures necessary to commence and maintain the enrichment over the specified period and beyond.

1. Assigning rats to enrichment cage groups

a. Rats should be designated to enrichment cage groups, and maintained in those groups throughout the enrichment period. Combining rats (or mice) from different (previous) standard cages is important, so that both some old and new cage-mates are now housed together to promote new social interactions. It is also important, however, that the standardhoused rodents are rehoused with new mates in a similar fashion to avoid differences between enriched and standard-housed mates in terms of this general manipulation. Note that some effects of enrichment may not be as strong when combining rats that have been raised individually (single-housed; Hellemans et al., 2004). For rats, raising them individually is not preferred, unless there is an explicit experimental question in mind. For example, there can be a relatively permanent increase in open field activity and poor inhibition after isolation rearing (Dalrymple-Alford and Benton, 1984). Our enrichment procedure has used 11-12 rats per enrichment cage. If much smaller or larger rats are being used, a smaller or larger enrichment cage could be used. However, the social interaction is more critical than the space afforded each rat within the cage for environmental enrichment so this is not strictly necessary. Cage size for enrichment can, of course, be adapted for use in mice (or younger rats), although the researcher should check the social interactions. Some male (house) mouse strains can be very aggressive, unlike most rat strains. Do not put rats together that have recently, for whatever reason, been interacting with opposite sex animals, as even male rats can then become aggressive.

b. It is important to establish method(s) to keep track of individual animals within the enrichment cages. Our rats had earpunch labels, but all animals also had individual tail markings made with a permanent marker and refreshed at least once weekly or sooner. These methods allowed us to keep track of animals for any return to standard housing, cage-cleaning, object changes, and behavioral testing.

c. If using environmental enrichment as a treatment model, we recommend combining control and experimental rats within each enrichment group and standard housing. This removes any possible unique injury- or treatment-related effect on the enrichment experience.

d. Multiple enrichment cages/groups can be run simultaneously by ensuring each different cage has a different order of templates within that cycle. This ensures that enrichment objects are only needed in one of the cages per day, and that cages all experience a similar sequence of enrichment days and the same sequence of cycles. Table 1 shows an example of how to run multiple enrichment cages with a different order of enrichment days but within the same cycle. 
Table 1. Method for running concurrent multiple enrichment cages. Enrichment days 140 are shown on subsequent rows. The numbers in the columns for Cage 1-Cage 4 specify the enrichment template to use for that cage on the given day.

\begin{tabular}{|c|c|c|c|c|}
\hline Day & Cage 1 & Cage 2 & Cage 3 & Cage 4 \\
\hline 1 & 1 & 2 & 3 & 4 \\
\hline 2 & 2 & 3 & 4 & 5 \\
\hline 3 & 3 & 4 & 5 & 6 \\
\hline 4 & 4 & 5 & 6 & 7 \\
\hline 5 & 5 & 6 & 7 & 1 \\
\hline 6 & 6 & 7 & 1 & 2 \\
\hline 7 & 7 & 1 & 2 & 3 \\
\hline 8 & 8 & 8 & 8 & 8 \\
\hline 9 & 9 & 10 & 11 & 12 \\
\hline 10 & 10 & 11 & 12 & 13 \\
\hline 11 & 11 & 12 & 13 & 14 \\
\hline 12 & 12 & 13 & 14 & 15 \\
\hline 13 & 13 & 14 & 15 & 9 \\
\hline 14 & 14 & 15 & 9 & 10 \\
\hline 15 & 15 & 9 & 10 & 11 \\
\hline 16 & 16 & 16 & 16 & 16 \\
\hline 17 & 17 & 18 & 19 & 20 \\
\hline 18 & 18 & 19 & 20 & 21 \\
\hline 19 & 19 & 20 & 21 & 22 \\
\hline 20 & 20 & 21 & 22 & 23 \\
\hline 21 & 21 & 22 & 23 & 17 \\
\hline 22 & 22 & 23 & 17 & 18 \\
\hline 23 & 23 & 17 & 18 & 19 \\
\hline 24 & 24 & 24 & 24 & 24 \\
\hline 25 & 25 & 26 & 27 & 28 \\
\hline 26 & 26 & 27 & 28 & 29 \\
\hline 27 & 27 & 28 & 29 & 30 \\
\hline 28 & 28 & 29 & 30 & 31 \\
\hline 29 & 29 & 30 & 31 & 25 \\
\hline 30 & 30 & 31 & 25 & 26 \\
\hline 31 & 31 & 25 & 26 & 27 \\
\hline 32 & 32 & 32 & 32 & 32 \\
\hline 33 & 33 & 34 & 35 & 36 \\
\hline 34 & 34 & 35 & 36 & 37 \\
\hline 35 & 35 & 36 & 37 & 38 \\
\hline 36 & 36 & 37 & 38 & 39 \\
\hline 37 & 37 & 38 & 39 & 33 \\
\hline 38 & 38 & 39 & 33 & 34 \\
\hline 39 & 39 & 33 & 34 & 35 \\
\hline 40 & 40 & 40 & 40 & 40 \\
\hline
\end{tabular}

Note: Days 8, 16, 24, 32, and 40 were "no object" days for our enrichment paradigm, so all four cages are the same for those days. 
2. Daily maintenance of enrichment novelty

a. Once per day, enrichment objects are removed and a new configuration is set up within the cages. Rats should first be removed from the enrichment cage and returned to their designated holding cages while the configuration is changed. This would usually be for only 10-30 min, when rats were food-restricted, they would be given their daily food at this time while in their holding cage groups. The used objects are removed from the cage. A new set of clean objects is set up within the cage in the configuration specified by the template for that day. The rats are then returned to the enrichment cage.

b. Water bottles and food hoppers should be refilled and then replaced in new positions within the cage.

c. Rats can be returned to the enrichment cage, and then the same process can be repeated with the next enrichment cage.

d. Once all cages have been changed, all the used objects should be washed in hot water and detergent and then left to dry overnight so that they can be used again as clean objects to be added to an enrichment cage the next day.

e. This process is repeated every day for the duration of the enrichment. Enrichment cages should be cleaned periodically (at least once a week), during which rats are placed in a holding cage while the sawdust bedding is replaced.

f. Move the location of the enrichment cage(s) every $x$ days. Our custom-made cages sat on large stainless-steel racks. Every four days we switched the position of the four trolleys in the colony room to give each enrichment cage varied lighting and views out of the cage in each direction. Alternatively, if possible, enrichment cages could be moved between colony rooms every set number of days to enhance the novelty of the surrounding environment.

g. Optional: Continue enrichment overnight-only after the all-day + all-night enrichment period. In our study, after the 40 days of continuous enrichment, rats were removed daily to their designated standard cages at $\sim 9$ am (lights now off; we use reversed lighting) to undergo spatial memory testing. At $\sim 5-6 \mathrm{pm}$ all rats were returned to their enrichment cages overnight. Each enrichment cage repeated the cycle of configurations shown in Table 1. Standard-housed control rats stayed in their standard cages throughout this period. This was done to sustain and/or maximize any cognitive enhancement past the continuous period of environmental enrichment.

\section{Data analysis}

This section includes an example of experimental results from Harland et al. (2014), obtained using this enrichment protocol. In this study, rats received acute neurotoxic brain lesions to the anterior thalamic nuclei, a nodal point in the extended hippocampal memory system (Dalrymple-Alford et al., 2015), or a "sham" control surgery in which no neurotoxin was delivered. After a recovery period, roughly half of the animals were placed in an enriched environment for 40 days, and the others were 
housed in standard caging for this period. This produced four groups: 1. Sham standard-housed rats (SH-Std), 2. Lesion Standard-housed rats (AT-Std), 3. Sham enriched rats (SH-Enr), 4. Lesion enriched rats (AT-Enr). Spatial memory was tested in a spatial memory task in an 8-arm radial maze (Figure 4A), previously shown to be sensitive to anterior thalamic nuclei lesions. The number of errors was assessed in the four groups over 35 days of testing ( 7 blocks of 5 days each; Figure 4B). There were significant main effects for both Surgery-type and Housing-type (Two-Way ANOVA, Surgery Effect, $F_{(1,41)}=49.94, P<0.001 ; d=1.86$, Housing Effect, $\left.F_{(1,41)}=26.52, P<0.001\right)$. Posthoc Newman-Keuls tests showed that sham enriched rats had significantly reduced errors compared to sham standard-housed rats $(P<0.05)$. Interestingly, lesion enriched rats had significantly reduced errors compared to lesion standard-housed rats $(P<0.001)$, returning them to a level of performance that did not differ from the sham standard-housed rats $(P>0.15)$. Coronal sections from the hippocampus of these animals were processed with a metallic Golgi-Cox stain and the density of dendritic spines per $10 \mu \mathrm{m}$ on the basal and apical arbors of CA1 pyramidal neurons was assessed (Figure 4C). The hippocampus is a brain region associated with spatial memory in rodents. There were significant main effects for Surgery-type and Housing-type for both basal and apical dendritic spines [see Figure 4D, Two-Way ANOVAs, Basal Spines (Surgery Effect, $F_{(1,41)}=104.50, P<0.001$; Housing Effect, $\left.F_{(1,41)}=69.37, P<0.001\right)$; Apical Spines (Surgery Effect, $F_{(1,41)}=43.87, P<0.001$; Housing Effect, $\left.\left.F_{(1,41)}=22.68, P<0.001\right)\right]$. Post-hoc Newman-Keuls tests of basal spine density showed that sham enriched rats had denser spines compared to sham standard-housed rats $(P<0.001)$. Lesion enriched rats had denser basal spines compared to lesion standard-housed rats $(P<0.001)$, comparable to the density observed in sham standard-housed rats $(P>0.15$; see Figure $4 \mathrm{E})$. Similarly, post hoc Newman-Keuls tests showed higher apical spine density in sham enriched rats compared to sham standard-housed rats $(P<0.001)$. Lesion enriched rats had denser apical spines compared to lesion standard-housed rats $(P<0.02)$, again similar to the density observed in sham standard-housed rats $(P>0.15)$. See Harland et al. (2014) for more details on these experiments. 

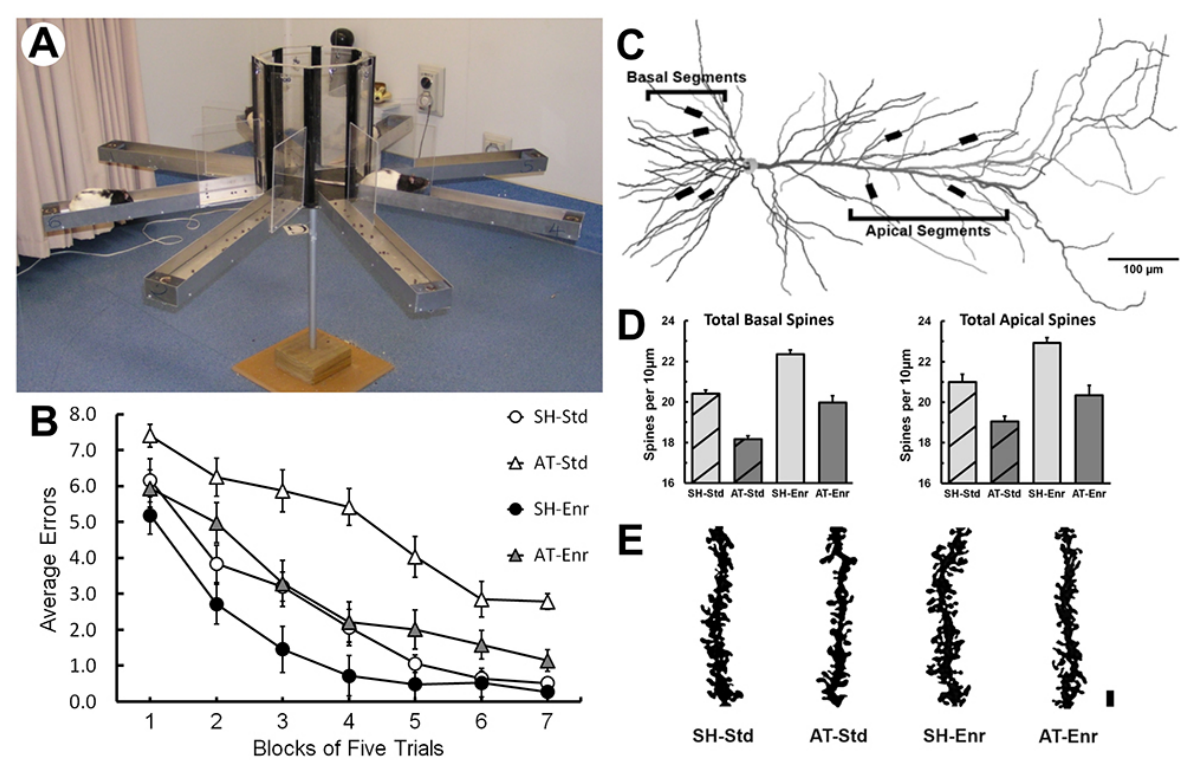

Figure 4. Examples of enrichment data. Adapted from Harland et al. (2014). A. Enrichment reduced the number of errors in a spatial memory task in a radial maze. B. Enriched rats that received a "sham" control surgery (SH-Enr) outperformed standard housed sham rats (SH-Std) in the radial-maze. Enriched rats with anterior thalamic nuclei lesions (AT-Enr) had reduced errors compared to standard-housed lesion rats performing the task at a comparable level to the standard-housed controls. C. Dendritic spine density was assessed along the basal and apical portions of CA1 pyramidal neurons in the hippocampus. D. Enrichment increased spine density in sham and lesion rats for both basal and apical dendritic spines. E. Representative overlaid projections of basal spine density are shown for each group, scale bar $=2 \mu \mathrm{m}$. Enrichment returned spine density in lesion rats to a similar level observed in sham standardhoused rats.

\section{Acknowledgments}

These protocols were designed for experiments published in Harland et al. (2014). We would like to thank Neroli Harris and Silvana De Freitas Costa for technical assistance. This work was supported by a Health Research Council of New Zealand Grant 09/051.

\section{Competing interests}

The authors declare no conflicts of interest or competing interests. 


\section{Ethics}

All procedures are in accordance with the $\mathrm{NIH}$ Guide for the Care and Use of Laboratory Animals and were approved by the University of Canterbury NZ Animal Ethics Committee ref 2015/21R (2015-2017).

\section{References}

1. Ali, M., Cholvin, T., Muller, M. A., Cosquer, B., Kelche, C., Cassel, J. C. and Pereira de Vasconcelos, A. (2017). Environmental enrichment enhances systems-level consolidation of a spatial memory after lesions of the ventral midline thalamus. Neurobiol Learn Mem 141: 108123.

2. Alwis, D. S. and Rajan, R. (2014). Environmental enrichment and the sensory brain: the role of enrichment in remediating brain injury. Front Syst Neurosci 8: 156.

3. Bilkey, D. K., Cheyne, K. R., Eckert, M. J., Lu, X., Chowdhury, S., Worley, P. F., Crandall, J. E. and Abraham, W. C. (2017). Exposure to complex environments results in more sparse representations of space in the hippocampus. Hippocampus 27(11): 1178-1191.

4. Bindu, B., Rekha, J. and Kutty, B. M. (2005). Post insult enriched housing improves the 8-arm radial maze performance but not the Morris water maze task in ventral subicular lesioned rats. Brain Res 1063(2): 121-131.

5. Bonaccorsi, J., Cintoli, S., Mastrogiacomo, R., Baldanzi, S., Braschi, C., Pizzorusso, T., Cenni, M. C. and Berardi, N. (2013). System consolidation of spatial memories in mice: effects of enriched environment. Neural Plast 2013: 956312.

6. Briones, T. L., Woods, J. and Rogozinska, M. (2013). Decreased neuroinflammation and increased brain energy homeostasis following environmental enrichment after mild traumatic brain injury is associated with improvement in cognitive function. Acta Neuropathol Commun 1: 57.

7. Cracchiolo, J. R., Mori, T., Nazian, S. J., Tan, J., Potter, H. and Arendash, G. W. (2007). Enhanced cognitive activity--over and above social or physical activity--is required to protect Alzheimer's mice against cognitive impairment, reduce Abeta deposition, and increase synaptic immunoreactivity. Neurobiol Learn Mem 88(3): 277-294.

8. Dalrymple-Alford, J. C. and Benton, D. (1984). Behavioural inhibition and the age at social isolation in rats. $Q J$ Exp Psychol 36(1B): 27-38.

9. Dalrymple-Alford, J. C., Harland, B., Loukavenko, E. A., Perry, B., Mercer, S., Collings, D. A., Ulrich, K., Abraham, W. C., McNaughton, N. and Wolff, M. (2015). Anterior thalamic nuclei lesions and recovery of function: Relevance to cognitive thalamus. Neurosci Biobehav Rev 54: 145-160.

10. Eckert, M. J. and Abraham, W. C. (2013). Effects of environmental enrichment exposure on synaptic transmission and plasticity in the hippocampus. Curr Top Behav Neurosci 15: 165-187. 
11. Einon, D. F., Morgan, M. J. and Will, B. E. (1980). Effects of post-operative environment onrecovery from dorsal hippocampal lesions in young rats: tests of spatial memory and motor transfer. Q J Exp Psychol 32: 137-148.

12. Gonzalez-Ramirez, M. M., Velazquez-Zamora, D. A., Olvera-Cortes, M. E. and GonzalezBurgos, I. (2014). Changes in the plastic properties of hippocampal dendritic spines underlie the attenuation of place learning in healthy aged rats. Neurobiol Learn Mem 109: 94-103.

13. Hannan, A. J. (2014). Environmental enrichment and brain repair: harnessing thetherapeutic effects of cognitive stimulation and physical activity to enhance experience-dependent plasticity. Neuropathol Appl Neurobiol 40: 13-25.

14. Harland, B. C., Collings, D. A., McNaughton, N., Abraham, W. C. and Dalrymple-Alford, J. C. (2014). Anterior thalamic lesions reduce spine density in both hippocampal CA1 and retrosplenial cortex, but enrichment rescues CA1 spines only. Hippocampus 24: 1232-1247.

15. Hellemans, K. G., Benge, L. C., Olmstead, M. C. (2004). Adolescent enrichment partially reverses the social isolation syndrome. Brain Res Brain Dev 150: 103-115.

16. Hirase, H. and Shinohara, Y. (2014). Transformation of cortical and hippocampal neural circuit by environmental enrichment. Neuroscience 280: 282-298.

17. Johansson, B. B. (2004). Functional and cellular effects of environmental enrichment after experimental brain infarcts. Restor Neurol Neurosci 22: 163-174.

18. Kempermann, G. (2019). Environmental enrichment, new neurons and the neurobiology of individuality. Nat Rev Neurosci 20: 235-245.

19. Kolb, B. and Gibb, R. (1991). Environmental enrichment and cortical injury: behavioral and anatomical consequences of frontal cortex lesions. Cereb. Cortex 1: 189-198.

20. Lawlor, M. (1990). The size of rodent cages. In Guidelines for the well-being of rodents in research (Guttman ed.). Bethesda: Scientist Centre for Animal Welfare p19-28.

21. Leger, M., Quiedeville, A., Paizanis, E., Natkunarajah, S., Freret, T., Boulouard, M. and Schumann-Bard, P. (2012). Environmental enrichment enhances episodic-likememory in association with a modified neuronal activation profile in adult mice. PloS One 7(10): e48043.

22. Makowska, I. J., Weary, D. M. (2016). The importance of burrowing, climbing and standing upright for laboratory rats. $R$ Soc Open Sci 3: 160136.

23. National Research Council (US). (2011). Guide for the Care and Use of Laboratory Animals. 8th edition. Washington (DC): National Academies Press (US). Available from: https://www.ncbi.nlm.nih.gov/books/NBK54050/ doi: 10.17226/12910.

24. Nithianantharajah, J. and Hannan, A.J. (2006). Enriched environments, experience-dependent plasticity and disorders of the nervous system. Nat Rev Neurosci 7(9): 697-709.

25. Ohline, S. M. and Abraham, W. C. (2019). Environmental enrichment effects on synaptic and cellular physiology of hippocampal neurons. Neuropharmacology 145: 3-12.

26. Olson, A. K., Eadie, B. D., Ernst, C. and Christie, B. R. (2006). Environmental enrichment and voluntary exercise massively increase neurogenesis in the adult hippocampus via dissociable pathways. Hippocampus 16: 250-260. 
Please cite this article as: Harland and Dalrymple-Alford, (2020). Enriched Environment Procedures for Rodents: Creating a Standardized Protocol for

Diverse Enrichment to Improve Consistency across Research Studies,Bio-protocol 10 (11): e3637. DOI: 10.21769/BioProtoc.3637.

27. Pang, T. Y. and Hannan, A. J. (2013). Enhancement of cognitive function in models of brain disease through environmental enrichment and physical activity. Neuropharmacology 64: 515528.

28. Rampon, C., Jiang, C. H., Dong, H., Tang, Y. P., Lockhart, D. J., Schultz, P. G., Tsien, J. Z. and $\mathrm{Hu}, \mathrm{Y}$. (2000a). Effects of environmental enrichment on gene expression in the brain. Proc Natl Acad Sci U S A 97: 12880-12884.

29. Rampon, C., Tang, Y. P., Goodhouse, J., Shimizu, E., Kyin, M. and Tsien, J. Z. (2000b). Enrichment induces structural changes and recovery from nonspatial memory deficits in CA1 NMDAR1-knockout mice. Nat Neurosci 3: 238-244.

30. Simpson, J. and Kelly, J. P. (2011). The impact of environmental enrichment in laboratoryratsbehavioural and neurochemical aspects. Behav Brain Res 222: 246-264.

31. Sozda, C. N., Hoffman, A. N., Olsen, A. S., Cheng, J. P., Zafonte, R. D. and Kline, A. E. (2010). Empirical comparison of typical and atypical environmental enrichment paradigms on functional and histological outcome after experimental traumatic brain injury. $J$ Neurotrauma 27: 10471057.

32. Tordoff, M. G., Alarcon, L. K., and Lawler, M. P. (2008). Preferences of 14 rat strains for 17 taste compounds. Physiol Behave 95(3): 308-332.

33. van Praag, H., Kempermann, G. and Gage, F. H. (2000). Neural consequences of environmental enrichment. Nat Rev Neurosci 1: 191-198.

34. Wallace, C. S., Kilman, V. L., Withers, G. S. and Greenough, W. T. (1992). Increases in dendritic length in occipital cortex after four days of differential housing in weanling rats. Behav Neural Biol 58: 64-68.

35. Will, B., Galani, R., Kelche, C. and Rosenzweig, M. R. (2004). Recovery from braininjury in animals: relative efficacy of environmental enrichment, physical exercise or formal training (1990-2002). Prog Neurobiol 72(3): 167-182.

36. Will, B., Toniolo, G., Kelche, C., Pallage, V., Deluzarche, F and Misslin, R. (1986). The effects of postoperative physical environment on novelty seeking behavior and maze learning in rats with hippocampal lesions. Behav Brain Res 19: 233-240.

37. Will, B. and Kelche, C. (1992). Environmental approaches to recovery of function from brain damage: a review of animal studies (1981 to 1991). Adv Exp Med Biol 325: 79-103. 